\title{
ARTICLE
}

\section{Multimodal neurocognitive markers of interoceptive tuning in smoked cocaine}

Alethia de la Fuente ${ }^{1,2}$, Lucas Sedeño ${ }^{1,2}$, Sofia Schurmann Vignaga ${ }^{1}$, Camila Ellmann ${ }^{1}$, Silvina Sonzogni ${ }^{2,3}$, Laura Belluscio ${ }^{2,3}$, Indira García-Cordero ${ }^{1,2}$, Eugenia Castagnaro ${ }^{1}$, Magdalena Boano ${ }^{1}$, Marcelo Cetkovich ${ }^{1}$, Teresa Torralva ${ }^{1}$, Eduardo T. Cánepa ${ }^{2,3}$, Enzo Tagliazucchi ${ }^{2,4}$, Adolfo M. Garcia ${ }^{1,2,5}$ and Agustín Ibañez $\mathbb{D}^{1,2,6,7,8}$

Contemporary neurocognitive models of drug addiction have associated this condition with changes in interoception - namely, the sensing and processing of body signals that fulfill homeostatic functions relevant for the onset and maintenance of addictive behavior. However, most previous evidence is inconsistent, behaviorally unspecific, and virtually null in terms of direct electrophysiological and multimodal markers. To circumvent these limitations, we conducted the first assessment of the relation between cardiac interoception and smoked cocaine dependence (SCD) in a sample of (a) 25 participants who fulfilled criteria for dependence on such a drug, (b) 22 participants addicted to insufflated clorhidrate cocaine (only for behavioral assessment), and (c) 25 healthy controls matched by age, gender, education, and socioeconomic status. We use a validated heartbeat-detection (HBD) task and measured modulations of the heart-evoked potential (HEP) during interoceptive accuracy and interoceptive learning conditions. We complemented this behavioral and electrophysiological data with offline structural (MRI) and functional connectivity (fMRI) analysis of the main interoceptive hubs. HBD and HEP results convergently showed that SCD subjects presented ongoing psychophysiological measures of enhanced interoceptive accuracy. This pattern was associated with a structural and functional tuning of interoceptive networks (reduced volume and specialized network segregation). Taken together, our findings provide the first evidence of an association between cardiac interoception and smoked cocaine, partially supporting models that propose hyper-interoception as a key aspect of addiction. More generally, our study shows that multimodal assessments of interoception could substantially inform the clinical and neurocognitive characterization of psychophysiological and neurocognitive adaptations triggered by addiction.

Neuropsychopharmacology (2019) 44:1425-1434; https://doi.org/10.1038/s41386-019-0370-3

\section{INTRODUCTION}

Drug addiction is a complex physiological and psychological phenomenon, characterized by an interplay of diverse biological mechanisms, such as alterations of the meso-limbic system. In particular, contemporary neurocognitive models have proposed that dopaminergic drug addiction may be crucially related to changes in interoception (namely, the sensing and processing of body signals) given its homeostatic role [1] in the onset and maintenance of addictive behavior [2]. Indeed, drug abusers and consumers present differential activation and/or functional connectivity (FC) patterns involving the insular cortex [3-6], a putative interoceptive hub [1]. However, extant results are inconclusive (showing both hypo- and hyper-reactivity/connectivity of the insular cortex) and virtually uninformed by relevant behavioral and multimodal neurocognitive measures. Against this background, we implemented a state-of-the-art multimodal approach combining behavioral, electrophysiological, volumetric, and FC markers to evaluate cardiac interoception in a sample of smoked cocaine dependents (SCD).

The development of addiction hinges on diverse neuroadaptative processes, particularly impacting on the reward system, the anti-reward system, and cognitive control mechanisms [7]. In addition, recent models propose that interoceptive experiences are critical for processing of sensory signals associated with pleasure and craving [8], two hallmarks of addiction. Moreover, in animal studies, anticipatory interoceptive markers such as cocaine peripheral effects over voltage channels [9] can serve as a peripheral interoceptive conditioned stimulus $[10,11]$ contributing to cocaine reward and cocaine seeking [11]. These results suggest that experience of interoceptive signals during consumption may facilitate associative learning between peripheral and rewarding effects. Therefore, sustained drug use may tune interoceptive pleasure-related mechanisms over time [8]. Repeated experiences over long periods are typically associated

\footnotetext{
${ }^{1}$ Institute of Cognitive and Translational Neuroscience (INCYT), INECO Foundation, Favaloro University, Buenos Aires, Argentina; ${ }^{2}$ National Scientific and Technical Research Council, Buenos Aires, Argentina; ${ }^{3}$ Laboratorio de Neuroepigenética, Departamento de Química Biológica, Facultad de Ciencias Exactas y Naturales, Universidad de Buenos Aires e IQUIBICEN, CONICET, Buenos Aires, Argentina; ${ }^{4}$ Buenos Aires Physics Institute (IFIBA) and Physics Department, University of Buenos Aires, Buenos Aires, Argentina; ${ }^{5}$ Faculty of Education, National University of Cuyo (UNCuyo), Mendoza M5502JMA, Argentina; ${ }^{6}$ Universidad Autónoma del Caribe, Barranquilla, Colombia; ${ }^{7}$ Center for Social and Cognitive Neuroscience, School of Psychology, Universidad Adolfo Ibáñez, Santiago, Chile and ${ }^{8}$ Australian Research Council, Centre of Excellence in Cognition and its Disorders, Macquarie University, Sydney, NSW, Australia

Correspondence: Agustín. Ibañez (aibanez@ineco.org.ar)
}

These authors contributed equally: Alethia de la Fuente, Lucas Sedeño

Received: 19 October 2018 Revised: 22 February 2019 Accepted: 2 March 2019

Published online: 13 March 2019 
to neuronal adaptations [12] and, during craving in addicted individuals, they could manifest as hyper-sensitivity to negative bodily sensations [13]. In this sense, previous research has linked interoceptive processing with insular modulations [3-6], including both hyper- [6] and hypo-connectivity [3]. In fact, healthy subjects trained to focus attention on the interoceptive domain exhibit modulations of the insula and associated neural systems [12], which may signal an allostatic adaptation following repeated consumption [7]. In brief, increased sensitivity to body-signals in drug abusers might lead to a neuroadaptation process similar to that observed for training in interoception [12] and other domains [14], characterized by reduced brain resources. Regarding structural signatures, two main processes seem to be potentially mixed while consumption took place during the synaptic pruning stage. First, gray matter volume reduction over the adolescence is related to performance, particularly over the frontal but also the insular cortex. Moreover, a negative association with performance has been reported for learning-related training [15-17], in line with the hypothesis of experience-dependent synaptic pruning [18]. Both lines of evidence suggest a developmental tuning. However, the links among behavioral correlates of interoceptive processing and related neurocognitive adaptations remain unexamined in substance use populations.

A relevant model to assess this issue is afforded by subjects addicted to a type of SC termed coca paste, the earliest intermediate product of clorhidrate cocaine (CC) extractions from the coca leaf. After chemical transformation, coca paste acquires low-melting points and can be volatilized and smoked [19]. While consumption of coca paste has been reported in the USA since the eighties [20], it currently represent a critical public health in developing countries. As observed by the Organization of American States (OAS) and by the United Nations Office on Drugs and Crime (UNODC), experimental research on its effects is scarce. Compared to CC, this basic form has earlier and stronger addictive properties [21], due to smoke inhalation as the administration route [22] and the presence of active adulterants [21, 22] and other coca alkaloids [23]. Such higher propensity to dependence [24] results in relatively poor therapeutic outcomes [25]. In particular, a drug's administration route is a critical determinant of addictiveness $[10,26]$. For instance, while insufflated cocaine boosts sympathetic peripheral signatures, SC engages dopaminergic reward systems more abruptly, facilitating contextual learning. Interoceptive cues related to repeated intravenous CC administration elicit dopaminergic responses, even when the central effects of cocaine are not present [10]. Thus, comparatively, $\mathrm{SC}$ is more likely to result in addiction, implying a strong sustained adaptation even after prolonged abstinence [25] in a range of systems, potentially including insular and interoceptive regions. Also, SC has been noted to induce aggressive behaviors [27], which, in turn, are associated with adaptations over insular and subcortical regions [28]. Moreover, changes in insulo-striatal connectivity have been related to impulsivity and relapse in SCdependent subjects [29]. Given the antecedents of stronger neuroadaptation, the basic previous results showing cocaine as a peripheral interoceptive conditioned stimulus, and aggressive behaviors in SC dependents, we hypothesized that this group would exhibit specific psychophysiological signatures of enhanced interoceptive processing, with a concomitant tuning of relevant neural regions and networks.

Although mainstream models postulate hyper-interoception in drug abusers, most results stem solely from (inconsistent) neuroimaging data [3-6] or the study of brain-lesioned patients [5], with only behavioral data from depressors such as alcohol, synthetic cannabinoids, or heroine [30-32], and almost null evidence on behavioral and electrophysiological interoceptive markers-such as the heart-evoked potential (HEP), an electrophysiological signature modulated by attention to interoceptive signals [33]. To bridge this gap, we assessed the relation between interoception and SC dependence with a novel multimodal and multidimensional approach [34]. We implemented a previously validated heartbeat detection (HBD) task [34], and measured ongoing HEP modulations as subjects tracked their own heartbeats in two conditions: first, without any external or internal cue (interoceptive accuracy $[I A])$, and then, after receiving auditory feedback (interoceptive learning [IL]). Moreover, using magnetic resonance imaging (MRI) and functional MRI (fMRI) data, we performed structural and FC analyses of the main interoceptive hubs associated with performance. Also, to test the specificity of behavioral SC-related effects on interoception, we administered the HBD task to demographically matched CC dependents (CCD) and healthy controls (CTR). Based on the above background, we advanced three main hypotheses. First, considering previous reports of increased anticipatory interoceptive signals in the fast administration route of cocaine, as well as the interoceptive hypothesis of addiction, we predicted that SCD subjects would exhibit enhanced IA relative to the CCD and CTR groups. Second, given that the HEP is usually modulated by interoceptive attention, we predicted that this enhanced performance in SCD would be accompanied by an enhanced psychophysiological signature of interoceptive processing (increased negative HEP modulation) in comparison with CTR subjects. Third, based on previous results on expertize and interoceptive training, we expected a specific tuning of interoceptive hubs (insular and related networks) in SCD subjects, at both morphological and functional levels.

\section{MATERIAL AND METHODS}

\section{Participants}

Seventy-two subjects were recruited in this study. Twenty-five participants fulfilled DSM-IV criteria for dependence to SCD, while 22 met criteria for CCD. All consumers had early onsets of drug consumption (between 14 and 16 years old, Table S2). As in similar reports [29, 35], some participants from both groups also presented dependence or abuse diagnosis for other drugs (Table S3). History of drug consumption was further evaluated with a comprehensive neuropsychiatric interview of lifetime substance use (Table S4), and with the Alcohol, Smoking, and Substance Involvement Screening Test (ASSIST) [36]: both SCD and CCD participants presented a significant preferential use of SC and CC, respectively (Tables S1 and S2)). Consumers presented no differences in current craving (CQC) [37], but SCD presented higher desire than CCD (Table S5) (see Supplementary Information 1 for further details).

We also included 25 healthy subjects (CTR) with no history of drug abuse (Tables S1 and S2), psychiatric or neurological disease (Table S1). All groups (SCD, CCD, and CTR) were age- and education-matched, and presented similar socio-economic statuses-as measured with the European Survey (ESOMAR) socioeconomic scale [38] and the Unsatisfied Basic Needs (UBN) scale [39] (Table 1). Groups showed no differences in heart rate (HR) and HR variability (Supplementary Information 1 and Table S6). All participants provided signed informed consent in accordance with the Declaration of Helsinki. The study protocol was approved by the institutional Ethics Committee ( $\mathrm{N}^{\circ} 609 / 16$, record 554).

\section{Behavioral assessment}

Interoceptive performance: $H B D$ task. We used a modified version of a validated HBD task [40] involving three conditions [34]. First, in the IA condition [41], participants were requested to follow their own heartbeats over 2 min via finger tapping, without any cue of their pulse from their neck or wrist. Second, in the IF condition, they performed the same task while receiving feedback via a stethoscope. Finally, in the IL condition [34], they were asked to follow their heartbeats again without any external cues (see Fig. 1, Supplementary Information 2 and Table S7 for further details). 
Table 1. Demography

\begin{tabular}{|c|c|c|c|c|c|c|c|}
\hline & $\mathrm{CTR}(N=25)$ & $\operatorname{SCD}(N=25)$ & $\operatorname{CCD}(N=22)$ & Statistics & & & \\
\hline Handedness (R:L) & 25:0 & $24: 1$ & 22:0 & $x^{2}=1.91, p=0.385$ & & & \\
\hline \multirow[t]{3}{*}{$\mathrm{BN}^{\mathrm{b}}$ (UBN: SBN:Street) } & 13:11:0 & $11: 5: 1$ & $5: 13: 0$ & $x^{2}=8.45, p=0.076$ & & & \\
\hline & & & & Model & \multicolumn{3}{|c|}{ Post hoc comparison (Tukey's HSD) } \\
\hline & & & & & CTR vs. SCD & CTR vs. CCD & SCD vs. CCD \\
\hline Education ${ }^{c}$ & $9.47(1.94)$ & $8.76(1.83)$ & $9.45(1.56)$ & $F(2)=2.027, p=0.139$ & n.s. & n.s. & n.s. \\
\hline Body mass index ${ }^{c}$ & $23.26(5.79)$ & $22.81(2.28)$ & 24.57 (3.47) & $F(2)=1.146, p=0.324$ & n.s. & n.s. & n.s. \\
\hline$A C E^{C}$ & $3.52(2.35)$ & $5.6(2.28)$ & $6.18(2.22)$ & $F(2)=10.440, p<0.001$ & 0.003 & 0.000 & 0.619 \\
\hline
\end{tabular}

Conceptually, the IA represents a basal interoceptive measures and IL reflects the integration of IF information to improve the performance (for more details see [34]). The total length of the HBD task was approximately $15 \mathrm{~min}$. During this task, electrocardiographic and high-density electroencephalographic (hd-EEG) signals were recorded, as detailed below.

As in a previous interoceptive study [42], we used a synchrony measure $\left(D_{\mathrm{m}}\right)$ to assess the participants' ability to adjust their responses to cardiac frequency changes over time. An advantage of this approach is that it is unbiased by the total number of responses-e.g., a subject who taps repeatedly may obtain high accuracy with other interoceptive indexes, even if the responses' oscillation is not adjusted to his/her heart-rate frequency [43]. $D_{\mathrm{m}}$ was calculated as the mean difference between motor-response frequency and cardiac frequency in each condition. This index is a positive score with a maximum of 0 , which indicates the smallest difference between answers and recorded heart beats and, thus, optimal interoceptive performance (see Fig. 1). As a majority of the subjects perform below $1 \mathrm{~Hz}$ of difference, and to simplify the interpretation of results, the measure is expressed as $1-D_{m}$ (see Supplementary Information 2 for details about the estimation of this measure).

\section{HEP acquisition and preprocessing}

During the HBD task, hd-EEG signals were recorded with a Biosemi Active-two 128-channel system, as in previous works [34] (see Supplementary Information 3 for further details). The resulting data were then segmented from $200 \mathrm{~ms}$ prior the R-wave-EKG onset to $800 \mathrm{~ms}$ after its onset. These EEG epochs were baseline-corrected relative to a -200 to $-0 \mathrm{~ms}$ time window and noisy epochs were rejected. EEG data from 50 participants (25 SCD and 25 CTR) complied with the requisites for adequate preprocessing analysis. The remaining number of cleaned epochs by condition did not differ across groups (Table S10). The HEP, which consists in a frontal deflection between 150 and $800 \mathrm{~ms}$ [33, 34], was measured around an extended frontal region of interest (ROI) encompassing nine electrodes [41]. We also assessed separate three-electrode ROls in frontal-right [67-69], frontal-left [81] and frontal-central topographies [41, 81], as previously reported [41].

MRI image acquisition and preprocessing

From the 72 participants (excluding the CCD participants who were only involved in the behavioral assessment), $43 \mathrm{MRI}$ recordings were used ( $20 \mathrm{SCD}$ and $23 \mathrm{CTR}$ )—omitted participants either abandoned the protocol or opted out of the MRI sessions due to claustrophobia. Both groups (SCD and CTR) were matched in age and education, and presented similar socio-economic statuses, as measured with the ESOMAR [44] and the UBN scale [39] (Table S11). Structural T1 scans and resting-state fMRI recordings were obtained through a $1.5 \mathrm{~T}$ Phillips Intera scanner with a head coil SENSE-NV phased array with eight channels. Resting-state was $10 \mathrm{~min}$ long, in witch participants were instructed not to think about anything in particular and keep their eyes closed (see Supplementary Information 4 for acquisition details). For structural images, Voxel-based morphometry (VBM) was performed to account for the structural correlates of interoceptive measures. Images were preprocessed with the CAT Toolbox from Statistical Parametric Mapping software (SPM12, see Supplementary Information 4 for preprocessing details). For functional image analysis, following previous FC reports [34], images were preprocessed with the Data Processing Assistant for Resting-State fMRI (DPARSF V4.3). Three SCD and two CTR subjects were excluded from the functional analysis due to excessive movement, resulting in a final sample of 18 SCD and 23 CTR participants (see Table S11). FC matrices were estimated using the Pearson's correlation coefficient and based on the Brainnetome Atlas [45].

Statistical analysis

Hypothesis 1: IA is enhanced in SCD subjects relative to CCD and CTR groups. To compare behavioral performance between groups, we used nonparametric Kruskal-Wallis tests (with Wilcoxon-Mann-Whitney tests for post hoc comparisons), reported with false-discovery rate (FDR), considered significant at $p$-FDR corrected $<0.05$. These tests were applied because data distribution depended on the condition blocks, visually evaluated by Q-Q plots and Shapiro-Wilk test for normality (see Supplementary Information 2). To make our results comparable with previous reports $[41,46,47]$, we excluded subjects as outliers for each condition when their outcomes were above 2.5 standard deviations (SDs) away from the group's mean. Note that this criterion allowed us to remove data that may not reflect the actual psychophysiological process targeted by the task while enhancing the power of the test to find truly significant results-as shown in simulation studies [48].

Finally, to explore the association between performance and consumption, we fit a random forest regression for subjects IA with consumption data. We reported mean $R^{2}$ over measured and predicted by consumption data, different to permutated subjects with $p<0.001$ (Mann-Whitney) (see Supplementary Information 2). 

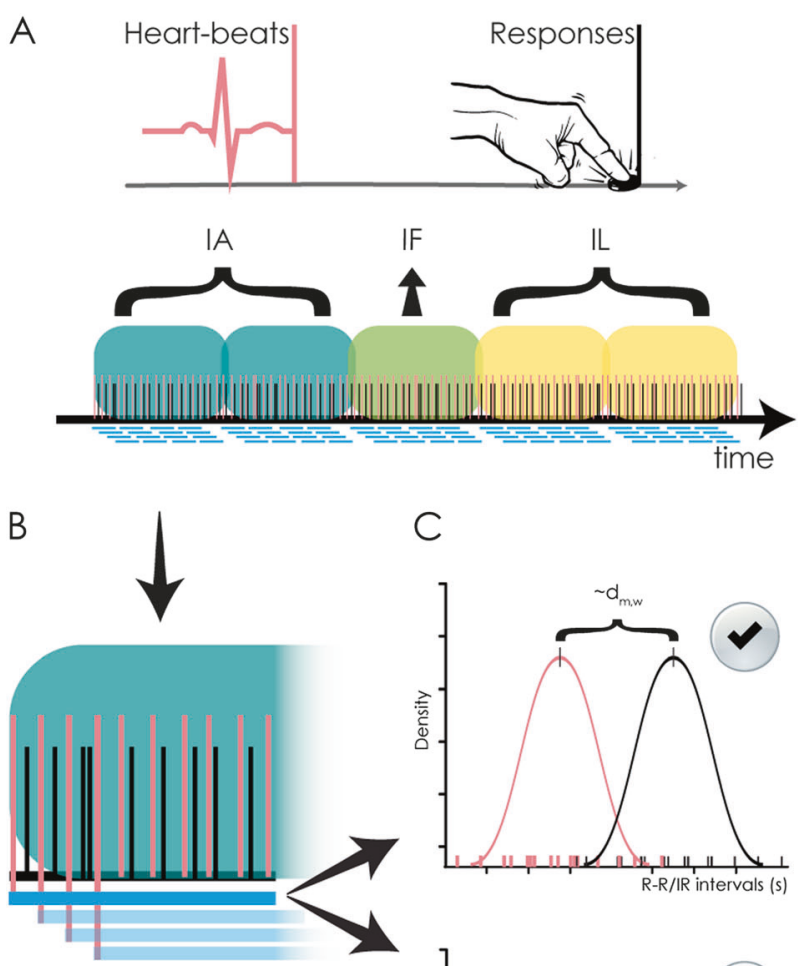

Individual window

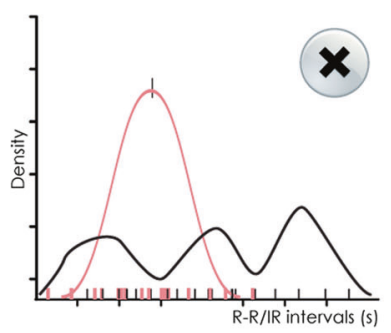

Fig. 1 Behavioral analysis. Performance was calculated using a smoothing moving average filter. a Over the task, heart beats (red) and responses (black) were registered and saved as marks in time. For each block (both IA, IF and both IL) the process was identical (dark green, IA; light green, IF; and yellow, IL). Each block was subsequently subdivided in overlapping windows starting at each single beat (blue lines). b Amplification of an individual window. Heart beats and responses are shown in red and black, respectively. In each window we computed inter heart-beats intervals $(R-R)$ and inter-responses intervals (IR intervals). c Inside each window the regularity of the responses was evaluated by the coefficient of variation (CV) to exclude extremely noisy windows. Panel $\mathbf{c}$ shows two illustrative examples of the distributions of R-R and IR intervals. Abscissa shows the expected density function and ordered the $R-R$ and IR intervals in seconds. Short lines in red and black illustrates individual RR and IR intervals in the window. While the regularity in the responses was enough (Up panel c) to assume the subjects were actually performing the task, the instant frequencies (window average frequency) of the heart (calculated as $1 /$ mean $R-R$ ) were compared with the instant frequency of responses (calculated as 1/ mean inter-response times). The absolute difference among heart and response frequencies were averaged among each window in the block, for each condition, to reveal the window specific $d_{m, w}$ for each condition (IA, IF, and IL). Responses with CV above 0.5 the window were discarded. IA interoceptive accuracy, IF interoceptive feedback, IL interoceptive learning

Hypothesis 2: enhanced performance in SCD subjects is accompanied by increased negative-HEP modulation in comparison with CTR subjects. As in previous cardiac interoception research [34], the HEP was compared between groups via a point-by-point Monte Carlo permutation test combined with bootstrapping in a $300-800 \mathrm{~ms}$ window- to avoid the influence of the cardiac field potential [34]. See Supplementary Information 3 for further details about data acquisition, preprocessing, and analysis.

Hypothesis 3: interoceptive hubs in SCD subjects are distinctively tuned at both morphological and functional levels. To establish the structural correlates of interoceptive measures for each group, we correlated VBM preprocessing results with the $D_{\mathrm{m}}$ from the IA and IL conditions via multiple regression analyses on SPM 12 [49]. Given that putative interoceptive areas correspond to frontal, temporal, and parietal regions [50], we used a mask to exclude occipital and cerebellar areas. Total intracranial volume was used as a covariate to discard the influence of brain-size differences (whole-brain analysis, $p<0.001$ uncorrected, extent threshold = 30 voxels [34]).

To examine the association between behavioral performance and FC, we focused our analysis on the connectivity between interoceptive areas and the rest of the brain. Core hubs from the interoceptive network were selected following previous reports [34], including the bilateral postcentral gyrus (regions 155, 156, 159, and 160 from the atlas), the insular cortex (regions 163 through 174), and the anterior cingulate cortex (ACC, regions 187 and 188) (Table S13). For this analysis, we used nonparametric spearman correlations ( $p<0.001$ uncorrected [34]).

Finally, to test for differences in the association of interoception and neuroimaging measures between groups, we implemented a linear regression to compare the relationship of interoceptive performance and total gray mater or FC of the associated clusters (for each SCD and CTR group). As in previous analyses, we excluded subjects whose gray matter, $F C$, or interoceptive performance outcomes fell 2.5 SDs away from the group's mean. We analyzed the interaction in the relationships of group to total gray mater or FC for CTRs and CSD ( $F$ test, $p<0.001$ ).

\section{RESULTS}

Interoceptive performance

We found an effect of IA across groups. Post hoc comparisons via Mann-Whitney and FDR correction showed that SCD performed better than CTR $(p=0.024)$ and CCD $(p=0.024)$. No group differences were found for either the IL condition of the interoceptive feedback condition (Fig. 2a, b, Table 2).

We found an association between poly-consumption data and IA $\left(R^{2}=0.108(0.013), p=0.032(0.012)\right.$, mean/SD values), different from subject permuted ( $p<0.001,1000$ permutations), mostly due SC amount and frequency (see Supplementary Information 2, Table S8 and Table S9 and Suppl. Fig 1).

\section{Heart-evoked potential}

A comparison of HEP modulations during IA between SCD and CTR revealed more negative deflections for the former group over the frontal ROI ( $p<0.05,5000$ permutations), within the expected time-window (650-750 ms, Fig. 3). No differences were found between groups in the IL condition in any ROI (Fig. 3 and Suppl. Fig. 2). Both IA and IL results were replicated in the frontal-left, frontal-right, and frontal-central ROls (Suppl. Fig. 2).

Association between interoceptive and neuroimaging measures Gray matter signatures of interoceptive performance. In CTR, IA positively correlated with gray matter volumes in the inferior frontal gyrus, the insulo-opercular region, and the left posterior cingulum $(p<0.001)$. In SCD, we observed a positive association between performance and the right frontal medial cortex, as well as negative association (i.e., higher IA correlated with decreased gray matter volume) with the insula, the left superior frontal gyrus, the bilateral putamen, and the left inferior parietal lobe $(p<0.001)$ (Fig. 4a and Table S12).

The IA condition revealed a significant interaction in the relationships of gray matter and groups for the left posterior 
cingulate gyrus $(p<0.001)$, the left superior temporal gyrus $(p<$ $0.001)$, the right putamen $(p<0.001)$, and the right insular cortex $(p<0.001)$. In all regions the relation was negative only for SCD (Table S12).

Significant associations also emerged for IL. In CTR, we performance was positively correlated with gray mater volume in several frontal areas, including the right frontal medial, precentral, frontal inferior orbital, insular, and frontal inferior opercular cortices; and the left frontal superior medial, frontal superior, frontal inferior pars orbitalis, and frontal medial

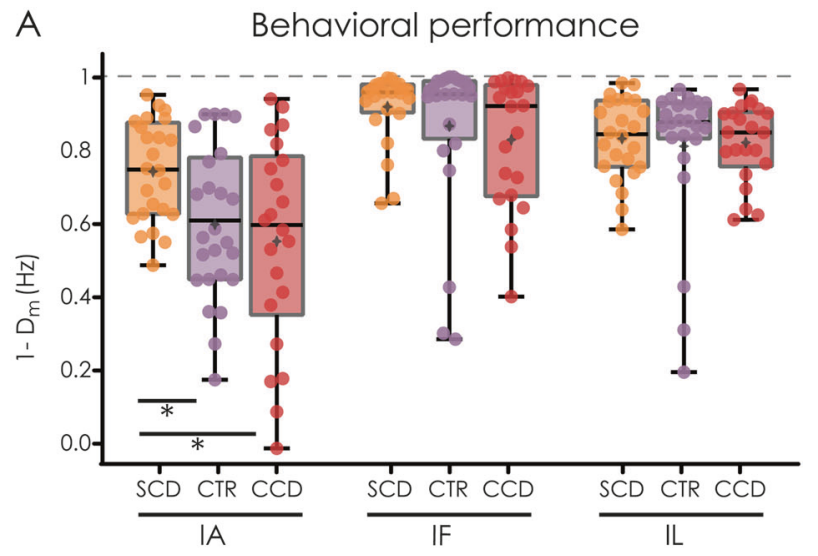

B

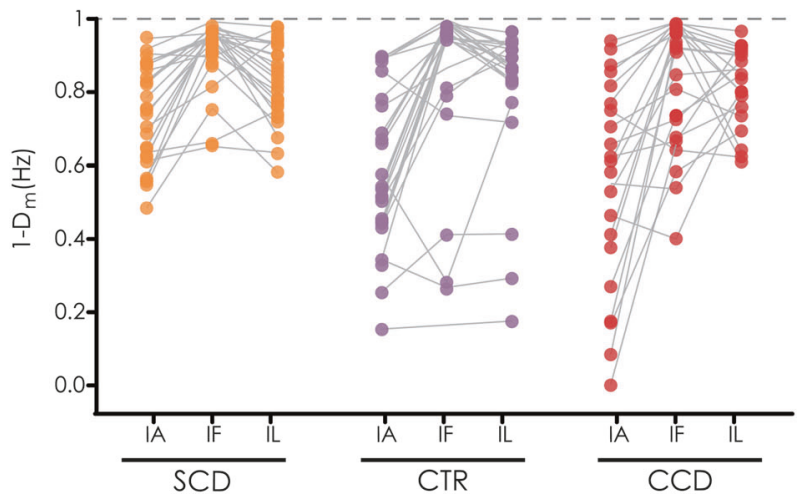

Fig. 2 Behavioral results. a Comparison of the performance of each group across conditions. SCD subjects has higher IA than both controls and CCD subjects. No differences were found for the interoceptive learning condition, nor for the feedback condition. Yellow indicates smoked cocaine dependent (SCD) subjects, violet indicates healthy controls (CTR), and red indicates CC dependent (CCD) subjects. The asterisk $\left(^{*}\right)$ indicates significant differences at a $p$-FDR corrected $<0.05$ ). b Changes of each subject's performance across conditions. Better outcomes are closer to 1. IA interoceptive accuracy, IF interoceptive feedback, IL interoceptive learning areas $(p<0.001)$. In SCD, IL was positively associated with the left frontal medial pars orbitalis, and negatively related to the right temporal superior and left supra marginal gyri $(p<0.001)$. The IL condition did not reveal any significant interaction in the relationship between gray matter and groups (Table S12) (Fig. 4a and Table S12).

Functional connectivity. In CTR, a positive association emerged between IA and FC between bilateral posterior insular and left postcentral regions with the precuneus, the posterior ventral cingulate gyrus, parietal regions, and the temporal pole $(p<0.001$, uncorrected). Conversely, in SCD, we found a negative association (i.e., better performance associated with decreased FC) involving connectivity of the bilateral anterior insular cortex with postcentral and temporal regions, as well as with posterior insular regions (Fig. 4b and Table S14). We found a significant interaction of group and performance in all relevant insula-related connections in SCD subjects. These links were found (a) between the left dorsolateral anterior insula and left superior posterior temporal sulcus $(p<0.001)$; (b) between the right dorsolateral posterior insula and the right medial temporal sulcus $(p<0.001)$, and (c) between the right dorsolateral anterior insula and three regions, namely: the left posterior superior temporal sulcus $(p<0.001)$, the left superior temporal sulcus $(p<0.001)$, and the left superior postcentral gyrus $(p<0.001)$; as well as between the right dorsolateral posterior insula and the right temporal medial sulcus $(p<.001)$. Among all regions the relation was negative only for SCD, details in Table S14.

In the IL condition, both groups presented negative associations between behavioral performance and the FC within insular areas, and between the latter and fronto-temporal regions $(p<0.001$, Fig. $4 \mathrm{~b}$ and Table S14). In the IL condition, we only found significant interactions of FC between the left dorsolateral anterior insula and the left posterior temporal sulcus $(p<0.001)$. This relation was negative for SCD — see details in Table S14.

\section{DISCUSSION}

Although neurocognitive models of drug addiction highlight the role of interoceptive processing in the onset and maintenance of addictive behavior [7], relevant multimodal evidence is scarce and controversial. Here, we report unprecedented multimodal neurocognitive evidence of enhanced cardiac interoception in addiction, showing that SCD subjects present boosts in relevant psychophysiological measures (HBD and HEP), accompanied by structural and FC tuning of interoceptive networks. More generally, our approach could inform the clinical and neurocognitive characterization of key biopsychological adaptations in addiction.

Psychophysiological evidence of enhanced ongoing interoception in SCD

SCD showed greater IA alongside enhanced negative modulations of the HEP, a physiological marker of attention to cardiac signals

\begin{tabular}{|c|c|c|c|c|c|c|c|}
\hline & CTR $(N=25)$ & $\mathrm{SCD}(N=25)$ & $C C D(N=22)$ & Model & CTR vs. SCD & CTR vs. CCD & SCD vs. $C C D$ \\
\hline IA & $0.410(0.208,25)$ & $0.266(0.138,24)$ & $0.457(0.280,22)$ & $\mathrm{KW}(3,71)=8.127, p=0.017$ & $p=0.024$ & n.s. & $p=0.024$ \\
\hline IF & $0.141(0.215,24)$ & $0.089(0.097,25)$ & $0.179(0.179,22)$ & $\mathrm{KW}(3,70)=2.104, p=0.349$ & n.s. & n.s. & n.s. \\
\hline IL & $0.197(0.204,24)$ & $0.176(0.110,25)$ & $0.187(0.106,22)$ & $\mathrm{KW}(3,70)=0.862, p=0.650$ & n.s. & n.s. & n.s. \\
\hline
\end{tabular}




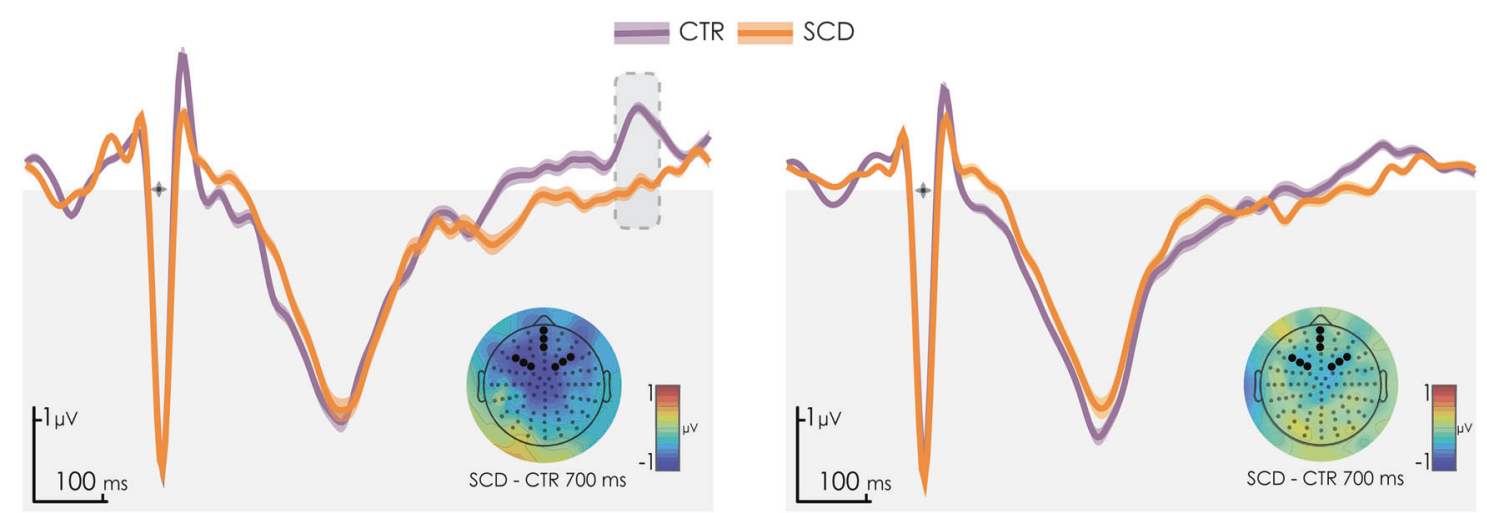

Fig. 3 Heart-evoked potential. HEP amplitude over the frontal region of interest (ROI). All differences reported were calculated via a point-bypoint Monte Carlo permutations analyses (5000 permutations, $p<0.05$ ). A differential modulation in IA emerged between 671 and 722 ms after R-wave. No differences were observed for the IL condition. A minimum extension of five consecutive points was selected as criterion to graph clusters, following previous reports [34]. Shadowed lines indicate SEM. Gray box indicate significant differences

$[33,40]$. Our results suggest that consumption (and, more particularly, the frequency and amount of SC consumption) impacts on IA. This finding supports the idea that sustained addiction critically hinges on increased interoceptive experiences during drug intake (as necessary for the pleasure states associated with consumption) and craving (given that abstinence involves negative body-sensations) [7]. Whereas relevant models have advanced compatible claims based on lesion studies and neuroimaging [3-6], our study extends such findings, for the first time, with evidence of ongoing behavioral and physiological measures of increased cardiac interoception in drug abusers.

More particularly, our results suggest that enhanced interoception may be associated to the specific drug or its route of administration. SCD subjects presented higher IA compared to the CCD group, which, in turn, was undifferentiated from healthy participants. Although SC and CC share the same active compound, differences in interoceptive processing may be related to the former's stronger addictive effect, which may amplify bodily sensations during consumption and craving [7]. SC includes adulterants (i.e., caffeine and phenacetine) that impact directly or indirectly [51] on the drug's rewarding effects [52]. Moreover, onset of acute drug effects depends on the consumption route: drug effects require a few seconds to manifest when smoked, but several minutes when insufflated [26]. This latency difference may affect the prediction of pleasurable bodily experiences, given that SCD consumers may expect a quicker effect and, hence, be more aware of body changes. Furthermore, anticipatory responses of body signals may trigger the reward system before the effect of a drug in the central nervous system [10]. The absence of statistical differences between CCD and CTR, though unexpected [8], may be also partly related to the relative less addictive power of CC as compared to SC. In this sense, the neuroadaptive process which drives hyper-interoceptive sensitivity in SC could be less marked than in CC. This may also impact the rehabilitation process of CCD subjects, with faster response to clinical treatments [25]. Moreover, previous research on CC has also shown inconclusive results [4], including decreases and increases of interoceptive network connectivity [53].

Regarding $\mathrm{IL}$, we did not find differences among SCD, CCD, and CTR. This may reflect a ceiling effect reached by the SCD group during the IA condition, given that their performance was not modulated by the information about their actual heart rate. Good IA performers do not increase accuracy after learning [40]. On the contrary, CCD and CTR subjects presented lower outcomes during $I A$, given that their baseline performance allowed for improvements after the feedback condition. Note that IL involves other regions (hippocampus and frontal cortex) and processes (memory formation and recovery) beyond interoception [34]. Also, these findings are consistent with the presence of HEP differences in IA but not in IL. Thus, our results suggest that SC impacts specifically on IA, namely, the core interoceptive dimension [54].

In sum, our findings provide partial support for the hyperinteroception hypothesis [3-6], by showing that this may be dependent on the type of drug and its associated features (e.g., administration route).

Interoceptive tuning of brain structures and FC in SCD At a structural level, in the CTR group, the relation between IA and fronto-insular structures replicated previous results [34]. In contrast, SCD showed a specific inverse association of IA with the volume of the right anterior insula (i.e., the lesser the volume and the better the performance). This inverted effect might have theoretical implications given that it resembles experience-based adaptations [55] and that consumption took place over the synaptic pruning stage [56] (Suppl. Discussion 5). Moreover, reduced gray matter volume over interoceptive areas has been observed in addiction [57], further suggesting a tuning process. Similarly, the putamen also presented the same inverse pattern in SCD. This supports previous evidence showing that both the putamen and the insula are associated with impulsivity and relapse in SCD [29]. Moreover, the interaction between groups and IA was significant for the core interoceptive areas (left posterior cingulum and right insular cortex) and also for the right putamen and the left superior temporal gyrus, always showing reduced gray matter for SCD subjects (Table S12).

Importantly, the interoceptive neurocognitive tuning in SCD subjects seems to be specific for core interoceptive processes. In fact, other regions related to less crucial interoceptive dimensions (i.e., learning, memory, and motor control) during IL showed no group differences in the direction of the association (Table S12) and replicated previous reports in controls (i.e., the larger the volume and the better the performance) [34]. This suggests that IL may not be affected by the neuroadaptive changes associated with drug consumption, which is expected given that IL relies on memory and learning skills [34]. However, superior temporal regions in the SCD group did still present an inverse pattern during IL. Although this is not a putative region of the interoceptive network, it has been related to increased performance in both IA and IL processes [34]. Therefore, its inverse direction during IL in the SCD is consistent with the direction of our results in the IA condition, which is associated with the neuroadaptive process affecting interoceptive networks. In this 

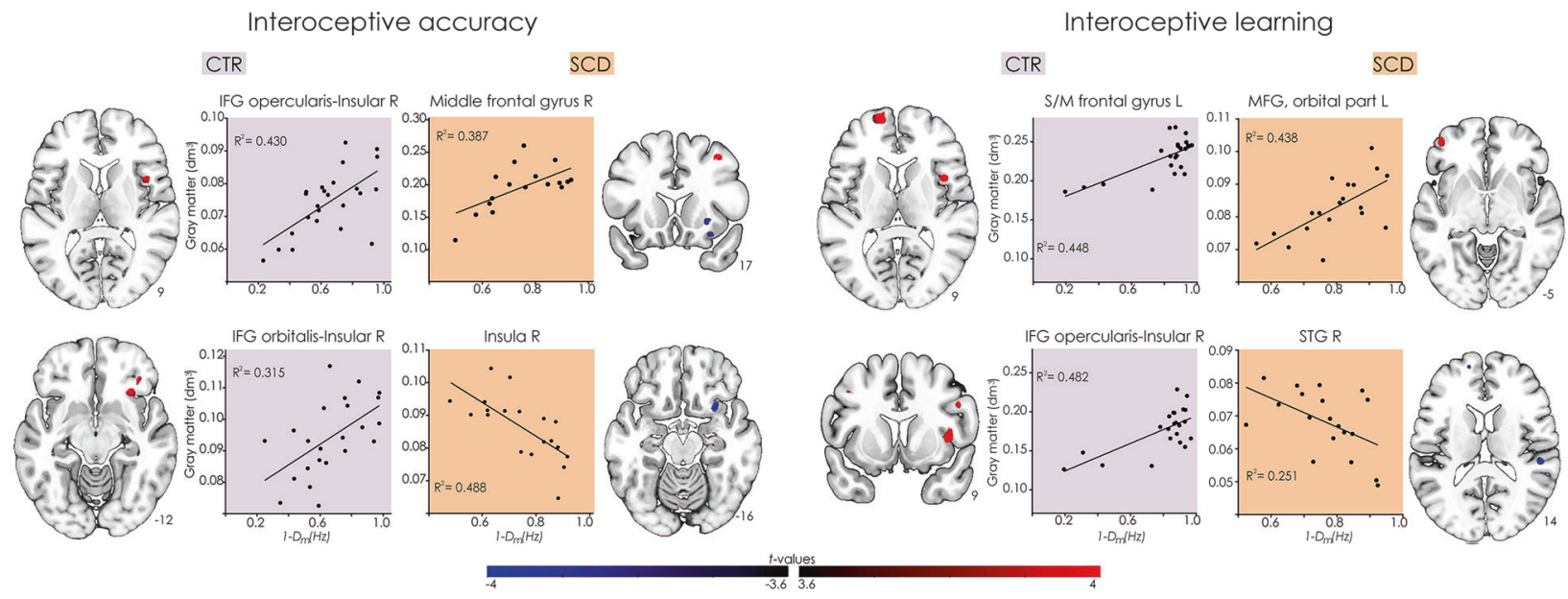

B

Functional connectvity

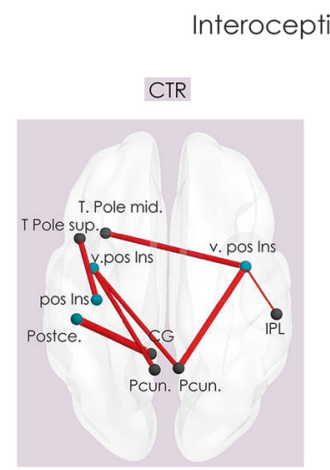

accuracy

\section{SCD}

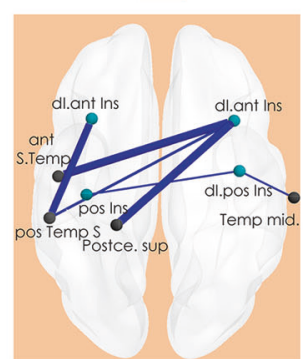
Positive association Interoceptive seeds
Negative association Other regions

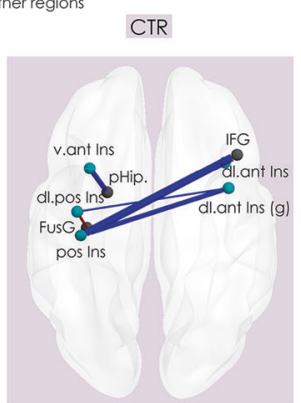

Interoceptive learning

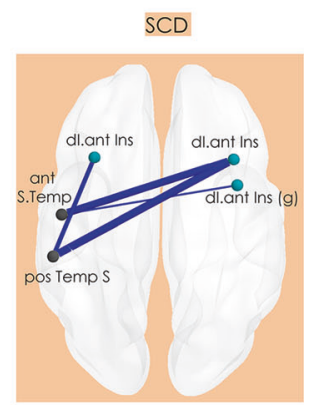

Fig. 4 Association between interoceptive performance and brain volume and functional connectivity. a Linear regression between gray matter volume and performance in the IA and IL conditions for the CTR and SCD groups. IA: in CTR, a positive association emerged between performance and gray matter volume in the inferior frontal gyrus, the pars opercularis-insula, the pars orbitalis-insula, and the left posterior cingulum; in SCD, a positive association was observed between performance and the right frontal medial cortex, alongside an inverse association with the left superior frontal gyrus, the insula, the bilateral putamen, and left parietal inferior areas. IL: in CTR, we found a positive association with the right frontal medial, precentral, frontal inferior orbital, insular, and frontal inferior opercular cortices; and the left frontal superior medial, frontal superior, frontal inferior pars orbitalis, and frontal medial areas; in SCD, a positive association emerged with the left frontal medial pars orbitalis, alongside a negative association with the right temporal superior and left supra marginal gyri. As in Fig. 1, better outcomes are closer to 1. Brain images are presented according to neurological convention. Scatterplots correspond to the most extensive clusters for each significant contrast (negative or positive relation between variables) per group (Supplementary Table 7). In the colormap, blue indicates negative associations between performance and gray matter volume, while red indicates positive ones. $\mathbf{b}$ Spearman correlation between performance and FC of the interoceptive network. IA: in CTR, a positive association was observed between performance and FC between the bilateral posterior insular and the postcentral left regions with the precuneus, posterior ventral cingulate gyrus, parietal regions, and the temporal pole; in SCD, we found a negative association of the bilateral anterior insular cortex with postcentral and temporal regions, and with posterior insular regions. IL: both groups presented negative associations between behavioral performance and the FC within insular areas, and between the latter and frontal and temporal regions. Cyan nodes represent main interoceptive regions, while areas outside them are depicted in dark gray (regions are from the Brainnetome [45]. The blue edges represent significant negative associations between performance and FC; while red edges indicate positive correlations. L: left; R: right; Brainnetome tags for areas: T. Pole sup.: lateral area $38 \mathrm{~L}$; pos Ins: hypergranular insula; Pcun: area 31 (LC1) ;v. pos Ins: ventral dysgranular and granular insula; T. Pole mid: medial area 38; IPL: rostroventral area 40(PFop); Postce: area 2;CG: ventral area 23; pos Temp S; area 41/42; dl. ant Ins: dorsal agranular insula; S. Temp: 0 and TE1.2; Postce. Sup: area1/2/3(trunk region); Temp mid.: caudal area 22; dl. pos Ins: dorsal granular insula; IFG: opercular area 44; pHip: area TI (temporal agranular insular cortex) ; v. ant Ins: ventral agranular insula; FusG: rostroventral area 20; dl. ant Ins (g): dorsal dysgranular insula

way, given that IL depends on both interoceptive and memory/ learning processes, it is expected that performance in the SCD may be directly associated with gray matter volume in memory/ learning-related areas, while, at the same time, with the inverse contribution of regions linked to the interoceptive network, such as superior temporal areas.

A similar pattern emerged in FC results. In CTR, IA was associated with the posterior insula (the primary cortical region receiving information from the thalamus to the insula) and its posterior interoception-related networks [58], as already reported by García-Cordero et al. [34]. By contrast, SCD subjects presented a negative association of the bilateral anterior insula (a secondary interoceptive portion of the insula, implicated in later processing and integrative functions) with (a) postcentral/temporal regions and (b) posterior insular regions, suggesting a neurocognitive tuning (i.e., the better the performance and the lower the FC), which is consistent with reports of network modulation induced by interoceptive training in interoceptive [12] and other domains [59] (Suppl. Discussion 5). Once again, this neurocognitive adaptation was observed for IA and restricted to anterior insular networks, a core hub of interoceptive information association. This result is consistent with evidence of different pleasant 
interoceptive stimulus [60], in which stimulant users show selective anterior insula responses. Interestingly, this effect has been found for pleasurable interoceptive stimuli also in consumption of depressors, such as alcohol and cannabis, but it is important to note that those groups also differed in their consumption of other drugs (such as stimulants) [61]. Thus, SCD performance may be reliant on the network independence and specialization of the anterior bilateral insular cortex, a region critical for top-down interoceptive processing [62] that proves sensitive to training [12]. Also, insular connectivity was differentially associated to performance between groups (Table S14). In the IL condition, enhanced performance was associated with a reduced association among fronto-temporo-insular networks in both CTR and SCD. This consistency among groups can be associated with the behavioral improvement of CTR subjects, whose post-training performance reached that of SCD subjects. This result is further supported by the lack of differences in IL slops (with the exception of the connection among temporal superior sulcus and insula, IA- and IL-related network) (Table S14). In this sense, note that feedback induces training and behavioral improvements in the HBD task [40], potentially associated with (short-term) network specializations (reduced connection of fronto-temporo-insular structures). In fact, acute training can lead to reductions in FC [63].

In sum, the structural and FC associations in IA support the hypothesis of enhanced interoception in SCD, associated with experience-based neural adaptations after sustained consumption [55]. Taken together, our results provide convergent evidence of a neurocognitive tuning of interoceptive areas in SCD, characterized by reduced resources and specialized network segregation. Compatibly, insular involvement has been interpreted by current models of addiction as driven by pleasant/unpleasant stimuli as well as craving and relapse. Here, we propose a complementary interpretation related to adaptation and expertize, consistent with the hyperinteroception expected as a result of an allostatic adaptation over the antireward system [7].

Limitations and future assessments

Our study has some limitations. First, our sample size was relatively small; however, it proved similar to those of prior studies [29]. Also, this caveat was counteracted by the strict control of several demographic, clinical, and substance-use-related variables, which have been scarcely controlled in previous reports [3-6]. Moreover, the analysis of convergent information from behavioral outcomes, EEG markers, and multimodal imaging signatures represents an additional source of robustness [34]. In line with previous studies [64], and considering our modest sample size, all neuroimaging results were reported with uncorrected statistical thresholds $(p<0.001$ uncorrected with 30 voxels as extent threshold for the voxel-wise analysis; $p<0.001$ uncorrected for FC associations with performance, as well as comparisons of VBM and FC slopes among groups). For the sake of consistency, associations between FC and behavior were performed with the same uncorrected threshold-and ensuing results aligned well with the putative role of the insula in interoception [34,65]. Similarly, the same threshold was employed for the lineal models to evaluate the interaction between the VBM and FC with behavior performance analysis [66-69]. Further research with large samples should confirm these patterns with more stringent criteria. Second, although our samples were matched by gender, most subjects enrolled were male. This is a limitation of most of addiction reports [35], given that, as shown by the UNODC, men have higher incidence of dependence on drugs, in general, and SC, in particular [70]. Third, SCD subjects have consumed a variety of other substances-which is a common behavior among consumers (4)-and this may have biased their interoceptive performance [5]. To overcome this limitation, we included a contrastive addiction model, CCD, that also presented an elevated consumption of several substances except for SC. This additional control approach is very scarce in drug research [3-6], and should be contemplated in future studies. Finally, additional experiments using similar ongoing neurocognitive markers of interoception should assess whether this enhancement in IA is also found before the onset of addiction (as a vulnerability marker) and when addiction starts during adulthood (given the decrease in neuroplasticity by the end of the adolescence [71]). Moreover, future investigations should explore whether greater IA is also associated with other substances and different consumption routes.

\section{CONCLUSIONS}

At the theoretical level, our results support the view that addictive behavior involves hyperinteroceptive processing [7]. Our multimodal approach shows that this interoceptive boost is associated with online psychophysiological modulations and offline brain adaptations, which resembles patterns of training-induced expertize. Analyzing addiction as a training process over interoceptive domain gives us a framework to understand its relation with the cues that facilitate relapse [72]. In this way, our report nurtures contemporary drug models with evidence about core interoceptive enhancement in drug consumption. Also, our findings open a new agenda for clinical settings. For instance, future studies may evaluate whether interoception is (i) related to specific symptoms in addiction [such as panic attacks or other anxiety disorders [73]], (ii) affected by different addiction stages, and (iii) distinctively modulated at initiation and maintenance or by the specific mechanisms of different drug types. Promisingly, this agenda would align current transnosological and dimensional approaches to interoception in psychiatric [74] and neurological [34] diseases. A dimensional approach which includes interoceptive processing may potentially contribute on the characterization, diagnosis, treatment, and prognosis of addiction. Finally, interventions that aim to modify body-signals perception and regulation, specifically mindfulness [75], may help to decrease the cues associated with the urge for consumption. However, these treatments should be considered after treatments for acute stressful [76-78] and vulnerable $[79,80]$ situations, which a priori represents a critical obstacle to overcome [81].

\section{FUNDING AND DISCLOSURE}

This work was partially supported by grants from CONICET, CONICYT/FONDECYT Regular (1170010), FONDAP 15150012, INECO Foundation, by the Inter-American Development Bank (IDB), and by PICT, Grant/Award number 2017-1818 and 20171820. PIP, Grant/Award number $9671 / 14$ and Foundation Florencio Perez. M. Cetkovich declares he has received monetary compensations as speaker from Gador, Lundbeck, Abbott, Pfizer, Baliarda, Roemmers, TEVA, Janssen, and Grunenthal in the last 3 years. The other authors declare no competing interests.

\section{ACKNOWLEDGEMENTS}

The authors acknowledge the Federación de Organizaciones no Gubernamentales de la Argentina para la Prevención y el Tratamiento de Abuso de Drogas (FONGA) as well as the patients, clinicians, and operators of the therapeutic communities (Buen Sanmaritano, Reparo, Foundation Creer es crear, Creando la libertad, Palomar, Modelo Minnesota). Also, we acknowledge the incredibly generous and committed help of the people of the nonprofit organizations who connected us with the control participants (Center Juan Pablo II, Foundation Temas, Matanza secretary, Agustin at Barrio Miter).

\section{ADDITIONAL INFORMATION}

Supplementary Information accompanies this paper at (https://doi.org/10.1038/ s41386-019-0370-3). 
Publisher's note: Springer Nature remains neutral with regard to jurisdictional claims in published maps and institutional affiliations.

\section{REFERENCES}

1. Craig $A D$. Interoception: the sense of the physiological condition of the body. Curr Opin Neurobiol. 2003;13:500-5. https://doi.org/10.1016/S0959-4388(03)00090-4

2. Koob GF, Le Moal M. Drug addiction, dysregulation of reward, and allostasis. Neuropsychopharmacology. 2001;24:97-129. https://doi.org/10.1016/S0893-133X (00)00195-0

3. Ding $X$, Lee S-W. Changes of functional and effective connectivity in smoking replenishment on deprived heavy smokers: a resting-state $\mathrm{fMRI}$ study. PLoS ONE. 2013;8:e59331 https://doi.org/10.1371/journal.pone.0059331

4. Ma L, Steinberg JL, Moeller FG, Johns SE, Narayana PA. Effect of cocaine dependence on brain connections: clinical implications. Expert Rev Neurother. 2015;15:1307-19. https://doi.org/10.1586/14737175.2015.1103183

5. Naqvi $\mathrm{NH}$, Rudrauf $\mathrm{D}$, Damasio $\mathrm{H}$, Bechara A. Damage to the insula disrupts addition to cigarette smoking. Science. 2007;315:531-5. https://doi.org/10.1126/ science.1135926.Damage

6. Sutherland MT, Carroll AJ, Salmeron BJ, Ross TJ, Stein EA Insula's functional connectivity with ventromedial prefrontal cortex mediates the impact of trait alexithymia on state tobacco craving. Psychopharmacology 2013;228: https://doi. org/10.1007/s00213-013-3018-8

7. Koob GF. Negative reinforcement in drug addiction: the darkness within. Curr Opin Neurobiol. 2013;23:559-63. https://doi.org/10.1016/j.conb.2013.03.011

8. Volkow ND, Morales M. The brain on drugs: from reward to addiction. Cell. 2015;162:712-25. https://doi.org/10.1016/j.cell.2015.07.046

9. Kiyatkin EA, Brown PLIv. cocaine induces rapid, transient excitation of striatal neurons via its action on peripheral neural elements: single-cell, iontophoretic study in awake and anesthetized rats. Neuroscience. 2007;148:978-95. https:// doi.org/10.1016/j.neuroscience.2007.07.013

10. Wise RA, Wang B, You Z-B. Cocaine serves as a peripheral interoceptive conditioned stimulus for central glutamate and dopamine release. PLoS ONE. 2008;3: e2846 https://doi.org/10.1371/journal.pone.0002846

11. Wang B, You Z-B, Oleson EB, Cheer JF, Myal S, Wise RA. Conditioned contribution of peripheral cocaine actions to cocaine reward and cocaine-seeking. Neuropsychopharmacology. 2013;38:1763 https://doi.org/10.1038/npp.2013.75

12. Cheng $Y$, Lin $C P$, Liu HL, Hsu YY, Lim KE, Hung D, et al. Expertise modulates the perception of pain in others. Curr Biol. 2007;17:1708-13. https://doi.org/10.1016/j. cub.2007.09.020

13. Goldstein RZ, Craig AD, Bechara A, Garavan H, Childress AR, Paulus MP, et al. The neurocircuitry of impaired insight in drug addiction. Trends Cogn Sci. 2009;13:372-80. https://doi.org/10.1016/j.tics.2009.06.004

14. Simmonds D, Luna B Protracted development of brain systems underlying working memory in adolescence: a longitudinal study. Neurolmage. https://doi. org/10.1016/j.neuroimage.2017.01.016 (2015).

15. Taubert M, Draganski B, Anwander A, Müller $K$, Horstmann A, Villringer $A$, et al. Dynamic properties of human brain structure: learning-related changes in cortical areas and associated fiber connections. J Neurosci. 2010;30:11670 https://doi.org/ 10.1523/JNEUROSCI.2567-10.2010

16. Elmer S, Hänggi J, Jäncke L. Processing demands upon cognitive, linguistic, and articulatory functions promote grey matter plasticity in the adult multilingual brain: Insights from simultaneous interpreters. Cortex. 2014;54:179-89. https:// doi.org/10.1016/j.cortex.2014.02.014

17. Zatorre RJ, Fields RD, Johansen-Berg H. Plasticity in gray and white: neuroimaging changes in brain structure during learning. Nat Neurosci. 2012;15:528 https://doi. org/10.1038/nn.3045

18. Ma L, Qiao Q, Tsai J-W, Yang G, Li W, Gan W-B. Experience-dependent plasticity of dendritic spines of layer 2/3 pyramidal neurons in the mouse cortex. Dev Neurobiol. 2016;76:277-86. https://doi.org/10.1002/dneu.22313

19. Magalhães EJ, Nascentes CC, Pereira LSA, Guedes MLO, Lordeiro RA, Auler LMLA, et al. Evaluation of the composition of street cocaine seized in two regions of Brazil. Sci Justice. 2013;53:425-32. https://doi.org/10.1016/j.scijus.2013.05.003

20. Kozel NJ. Reports of coca paste smoking: field investigations in South Florida and New York. Rockville: National Institute on Drug Abuse, Division of Epidemiology and Statistical Analysis; 1985.

21. Prieto JP, Galvalisi M, López-Hill X, Meikle MN, Abin-Carriquiry JA, Scorza C Caffeine enhances and accelerates the expression of sensitization induced by coca paste indicating its relevance as a main adulterant. Am J Addict. 2015;24:475-81. https://doi.org/10.1111/ajad.12245

22. Galvalisi M, Prieto JP, Martínez M, Abin-Carriquiry JA, Scorza C. Caffeine induces a stimulant effect and increases dopamine release in the nucleus accumbens shell through the pulmonary inhalation route of administration in rats. Neurotox Res. 2017;31:90-8. https://doi.org/10.1007/s12640-016-9667-8
23. EISohly MA, Brenneisen R, Jones AB. Coca paste: chemical analysis and smoking experiments. J Forensic Sci. 1991;36:93-103.

24. Navarro R, Yupanqui M, Geng J, Valdivia G, Girón M, Rojas M, et al. Development of a program of behavior modification directed to the rehabilitation of drugdependent patients: treatment and follow-up of 223 cases. Int J Addict. 1992;27:391-408. https://doi.org/10.3109/10826089209068749

25. Kiluk BD, Babuscio TA, Nich C, Carroll KM. Smokers versus snorters: do treatment outcomes differ according to route of cocaine administration? Exp Clin Psychopharmacol. 2013;21:490-8. https://doi.org/10.1037/a0034173

26. Samaha AN, Robinson TE. Why does the rapid delivery of drugs to the brain promote addiction? Trends Pharmacol Sci. 2005;26:82-7. https://doi.org/10.1016/ j.tips.2004.12.007

27. Davis WM. Psychopharmacologic violence associated with cocaine abuse kindling of a limbic dyscontrol syndrome? Progress Neuropsychopharmaco Biol Psychiatry. 1996;20:1273-300. https://doi.org/10.1016/S0278-5846(96) 00126-1

28. Khalsa SS, Adolphs R, Cameron OG, Critchley HD, Davenport PW, Feinstein JS, et al. Interoception and Mental Health: A Roadmap. Biological psychiatry. Cogn Neurosci neuroimaging. 2018;3:501-13. https://doi.org/10.1016/j. bpsc.2017.12.004

29. McHugh MJ, Demers CH, Braud J, Briggs R, Adinoff B, Stein EA. Striatal-insula circuits in cocaine addiction: implications for impulsivity and relapse risk. Am J Drug Alcohol Abus. 2013;39:424-32. https://doi.org/10.3109/00952990.2013.847446

30. Sönmez MB, Kahyacı Kılıç E, Ateş Çöl I, Görgülü Y, Köse Çınar R. Decreased interoceptive awareness in patients with substance use disorders. J Subst Use. 2017;22:60-5. https://doi.org/10.3109/14659891.2016.1143048

31. Ateş Çöl I, Sönmez MB, Vardar ME. Evaluation of Interoceptive awareness in alcohol-addicted patients. Noro Psikiyatr arsivi. 2016;53:17-22. https://doi.org/ 10.5152/npa.2015.9898

32. Schmidt AF, Eulenbruch T, Langer $C$, Banger M. Interoceptive awareness, tension reduction expectancies and self-reported drinking behavior. Alcohol Alcohol. 2013;48:472-7. https://doi.org/10.1093/alcalc/agt024

33. Yoris A, Abrevaya S, Esteves S, Salamone P, Lori N, Martorell M, et al. Multilevel convergence of interoceptive impairments in hypertension: new evidence of disrupted body-brain interactions. Hum Brain Mapp. 2018;39:1563-81. https:// doi.org/10.1002/hbm.23933

34. García-Cordero I, Sedeño L, de la Fuente L, Slachevsky A, Forno G, Klein F, et al. Feeling, learning from and being aware of inner states: interoceptive dimensions in neurodegeneration and stroke. Philos Trans $\mathrm{R}$ Soc Lond B Biol Sci. 1708:2016:371 https://doi.org/10.1098/rstb.2016.0006. 20160006

35. Adinoff B, Gu H, Merrick C, McHugh M, Jeon-Slaughter H, Lu H, et al. Basal hippocampal activity and its functional connectivity predicts cocaine relapse. Biol Psychiatry. 2015;78:1-9. https://doi.org/10.1016/j.biopsych.2014.12.027

36. Group WHOAW. The Alcohol, Smoking and Substance Involvement Screening Test (ASSIST): development, reliability and feasibility. Addiction. 2002;97:1183-94. https://doi.org/10.1046/j.1360-0443.2002.00185.x

37. Tiffany ST, Singleton E, Haertzen CA, Henningfield JE. The development of a cocaine craving questionnaire. Drug Alcohol Depend. 1993;34:19-28. https://doi. org/10.1016/0376-8716(93)90042-O

38. Liberona Y, Castillo O, Engler V, Villarroel L, Rozowski J. Nutritional profile of schoolchildren from different socio-economic levels in Santiago, Chile. Public Health Nutr. 2011;14:142-9. https://doi.org/10.1017/S1368980010001540

39. Pena R, Wall S, Persson LA. The effect of poverty, social inequity, and maternal education on infant mortality in Nicaragua, 1988-1993. Am J Public Health. 2000;90:64-9.

40. Canales-Johnson A, Silva C, Huepe D, Garcia M, Silva W, Ciraolo C, et al. Auditory feedback differentially modulates behavioral and neural markers of objective and subjective performance when tapping to your heartbeat. Cereb Cortex. https:// doi.org/10.1093/biostatistics/manuscript-acf-v5; 2015.

41. García-Cordero I, Esteves S, Mikulan EP, Hesse E, Baglivo FH, Silva W, et al. Attention, in and out: scalp-level and intracranial EEG correlates of interoception and exteroception. Front Neurosci. https://doi.org/10.3389/fnins.2017.00411; 2017.

42. Couto B, Salles A, Sedeno L, Peradejordi M, Barttfeld P, Canales-Johnson A, et al. The man who feels two hearts: the different pathways of interoception. Soc Cogn Affect Neurosci. 2014;9:1253-60. https://doi.org/10.1093/scan/nst108

43. Werner G, Mountcastle VB. The variability of central neural activity in a sensory system, and its implications for the central reflection of sensory events. J Neurophysiol. 1963;26:958-77. https://doi.org/10.1152/jn.1963.26.6.958

44. Hoffmeyer-Zlotnik JHP, Wolf C. ESOMAR European Society for Opinion and Marketing Research. The ESOMAR Standard Demographic Classification. Boston, MA: Springer US; 2003. p. 97-121.

45. Fan L, Li H, Zhuo J, Zhang Y, Wang J, Chen L, et al. The human brainnetome atlas: a new brain atlas based on connectional architecture. Cereb Cortex. 2016;26:3508-26. https://doi.org/10.1093/cercor/bhw157 
46. Couto B, Adolfi F, Sedeño L, Salles A, Canales-Johnson A, Alvarez-Abut $P$, et al. Disentangling interoception: insights from focal strokes affecting the perception of external and internal milieus. Front Psychol. 2015;6. https://doi.org/10.3389/ fpsyg.2015.00503

47. Couto B, Salles A, Sedeño L, Peradejordi M, Barttfeld P, Canales-Johnson A, et al. The man who feels two hearts: the different pathways of interoception. Soc Cogn Affect Neurosci. 2013;54:1-8. https://doi.org/10.1093/scan/nst108

48. Zimmerman DW. Increasing thE Power of Nonparametric Tests by Detecting and Downweighting Outliers. J Exp Educ. 1995;64:71-8.

49. Friston K, Ashburner J, Kiebel S, Nichols T, Penny W. Statistical Parametric Mapping (SPM), Academic Press; 2007. https://doi.org/10.1016/B978-012372560-8/ 50000-0

50. Pollatos $\mathrm{O}$, Herbert BM, Mai S, Kammer T. Changes in interoceptive processes following brain stimulation. Philos Trans R Soc Lond. 2016;371. https://doi.org/ 10.1098/rstb.2016.0016

51. Scorza C, Piccini C, Martínez Busi M, Abin Carriquiry JA, Zunino P. Alterations in the gut microbiota of rats chronically exposed to volatilized cocaine and its active adulterants caffeine and phenacetin. Neurotox Res. https://doi.org/10.1007/ s12640-018-9936-9; 2018.

52. Kiraly DD, Walker DM, Calipari ES, Labonte B, Issler O, Pena CJ, et al. Alterations of the host microbiome affect behavioral responses to cocaine. Sci Rep. 2016;6:35455 https://doi.org/10.1038/srep35455

53. Cisler JM, Elton A, Kennedy AP, Young J, Smitherman S, James GA, et al. Altered functional connectivity of the insular cortex across prefrontal networks in cocaine addiction. Psychiatry Res. 2013;213:39-46. https://doi.org/10.1016/j. pscychresns.2013.02.007

54. Garfinkel SN, Eccles JA, Critchley HD. The heart, the brain, and the regulation of emotion. JAMA Psychiatry. 2015;72:1071 https://doi.org/10.1001/ jamapsychiatry.2015.1493

55. Jeon HA, Friederici AD. What does "Being an Expert" mean to the brain? Functional specificity and connectivity in expertise. Cereb Cortex. 2017;27:5603-15. https://doi.org/10.1093/cercor/bhw329

56. Pfefferbaum A, Rohl T, Pohl KM, Lane B, Chu W, Kwon D, et al. Adolescent development of cortical and white matter structure in the NCANDA sample: role of sex, ethnicity, puberty, and alcohol drinking. Cereb Cortex. 2015;i:1-21. https:// doi.org/10.1093/cercor/bhv205

57. Franklin TR, Acton PD, Maldjian JA, Gray JD, Croft JR, Dackis CA, et al. Decreased gray matter concentration in the insular, orbitofrontal, cingulate, and temporal cortices of cocaine patients. Biol Psychiatry. 2002;51:134-42. https://doi.org/ 10.1016/S0006-3223(01)01269-0

58. Craig aD. A new view of pain as a homeostatic emotion. Trends Neurosci. 2003;26:303-7. https://doi.org/10.1016/S0166-2236(03)00123-1

59. Duan X, Liao W, Liang D, Qiu L, Gao Q, Liu C, et al. Large-scale brain networks in board game experts: insights from a domain-related task and task-free resting state. PLoS ONE. 2012;7:e32532 https://doi.org/10.1371/journal.pone.0032532

60. Stewart JL, May AC, Tapert SF, Paulus MP. Hyperactivation to pleasant interoceptive stimuli characterizes the transition to stimulant addiction. Drug Alcohol Depend. 2015;154:264-70. https://doi.org/10.1016/j.drugalcdep.2015.07.009

61. Migliorini R, Stewart JL, May AC, Tapert SF, Paulus MP. What do you feel? Adolescent drug and alcohol users show altered brain response to pleasant interoceptive stimuli. Drug Alcohol Depend. 2013;133:661-8. https://doi.org/10.1016/j. drugalcdep.2013.08.015

62. Murphy J, Brewer R, Catmur C, Bird G. Interoception and psychopathology: a developmental neuroscience perspective. Dev Cogn Neurosci. 2017;23:45-56. https://doi.org/10.1016/j.den.2016.12.006
63. Kelly AMC, Garavan H. Human functional neuroimaging of brain changes associated with practice. Cereb Cortex. 2005;15:1089-102. https://doi.org/10.1093/ cercor/bhi005

64. Sedeno L, Piguet O, Abrevaya S, Desmaras H, Garcia-Cordero I, Baez S, et al. Tackling variability: a multicenter study to provide a gold-standard network approach for frontotemporal dementia. Hum Brain Mapp. 2017;38:3804-22. https://doi.org/10.1002/hbm.23627

65. Moguilner S, García AM, Mikulan E, Hesse E, García-Cordero I, Melloni M, et al. Weighted Symbolic Dependence Metric (WSDM) for fMRI resting-state connectivity: a multicentric validation for frontotemporal dementia. Sci Rep. 2018;8:11181 https://doi.org/10.1038/s41598-018-29538-9

66. Abrevaya S, Sedeno L, Fitipaldi S, Pineda D, Lopera F, Buritica O, et al. The road less traveled: alternative pathways for action-verb processing in Parkinson's disease. J Alzheimers Dis. 2017;55:1429-35. https://doi.org/10.3233/JAD-160737

67. Mayer AR, Mannell MV, Ling J, Gasparovic C, Yeo RA. Functional connectivity in mild traumatic brain injury. Hum Brain Mapp. 2011;32:1825-35.

68. Wu T, Long $X$, Wang $L$, Hallett $M$, Zang $Y$, Li K, et al. Functional connectivity of cortical motor areas in the resting state in Parkinson's disease. Hum Brain Mapp. 2011;32:1443-57.

69. Wu X, Li R, Fleisher AS, Reiman EM, Guan X, Zhang Y, et al. Altered default mode network connectivity in Alzheimer's disease-a resting functional MRI and Bayesian network study. Hum Brain Mapp. 2011;32:1868-81.

70. Romo-Avilés N, Camarotti AC, Tarragona A, Touris C. Doing gender in a toxic world. Women and Freebase Cocaine in the City of Buenos Aires (Argentina). Subst Use Misuse. 2015;50:557-65. https://doi.org/10.3109/10826084.2014.991404

71. Grant BF, Dawson DA. Age of onset of drug use and its association with DSM-IV drug abuse and dependence: Results from the national longitudinal alcohol epidemiologic survey. J Subst Abus. 1998;10:163-73. https://doi.org/10.1016/ S0899-3289(99)80131-X

72. Gray MA, Critchley HD. Interoceptive basis to craving. Neuron. 2007;54:183-6. https://doi.org/10.1016/j.neuron.2007.03.024

73. Luthi $A$, Luscher $C$. Pathological circuit function underlying addiction and anxiety disorders. Nat Neurosci. 2014;17:1635-43. https://doi.org/10.1038/nn.3849

74. Khalsa SS, Lapidus RC. Can interoception improve the pragmatic search for biomarkers in psychiatry? Front Psychiatry. 2016;7:1-19. https://doi.org/10.3389/ fpsyt.2016.00121

75. Paulus MP, Stewart JL, Haase L. Treatment approaches for interoceptive dysfunctions in drug addiction. Front Psychiatry. 2013;4:137 https://doi.org/10.3389/ fpsyt.2013.00137

76. Sinha R, Jastreboff AM. Stress as a common risk factor for obesity and addiction. Biol Psychiatry. 2013;73:827-35. https://doi.org/10.1016/j.biopsych.2013.01.032

77. Martinez D, Orlowska D, Narendran R, Slifstein M, Liu F, Kumar D, et al. Dopamine type $2 / 3$ Receptor availability in the striatum and social status in human volunteers. Biol Psychiatry. 2010;67:275-8. https://doi.org/10.1016/j.biopsych.2009.07.037

78. Sinha R. The role of stress in addiction relapse. Curr Psychiatry Rep. 2007;9:388-95. https://doi.org/10.1007/s11920-007-0050-6

79. Seo D, Tsou Ka, Ansell EB, Potenza MN, Rajita S. Cumulative adversity sensitizes neural response to acute stress: association with health symptoms. Neuropsychopharmacology. 2014;39:670-80. https://doi.org/10.1038/npp.2013.250

80. Harrington M, Robinson J, Bolton SL, Sareen J, Bolton JM. A longitudinal study of risk factors for incident drug use in adults: Findings from a representative sample of the US population. Can J Psychiatry. 2011;56:686-95. https://doi.org/10.1177/ 070674371105601107

81. Schulz A, Vögele C. Interoception and stress. Front Psychol. 2015;6:993 https:// doi.org/10.3389/fpsyg.2015.00993 